\title{
Jatupalathika herbal formula inhibits lipid accumulation and induces lipolysis in 3T3-L1 adipocytes
}

\author{
Aurasorn Saraphanchotiwitthaya ${ }^{a, b, *}$, Pattana Sripalakit ${ }^{c}$ \\ a Department of Pharmaceutical Technology, Faculty of Pharmaceutical Sciences, Naresuan University, Phitsanulok \\ 65000 Thailand \\ b Pharmaceutical Biotechnology Research Unit, Faculty of Pharmaceutical Sciences, Naresuan University, Phitsanulok \\ 65000 Thailand \\ c Department of Pharmaceutical Chemistry and Pharmacognosy, Faculty of Pharmaceutical Sciences, Naresuan \\ University, Phitsanulok 65000 Thailand
}

*Corresponding author, e-mail: aurasorns@nu.ac.th

ABSTRACT: Jatupalathika is composed of four dried fruit of Terminalia bellerica (Gaertn.) Roxb, Terminalia chebula Retz. var. chebula, Terminalia arjuna Roxb., and Phyllanthus emblica L. This research aimed to investigate the antiobesity potential of Jatupalathika aqueous extracts on the inhibition of fat accumulation and the induction of lipolysis in 3T3-L1 adipocytes, compared with individual herbs in the formula, gallic acid, and ellagic acid at a series of concentrations of 10-100 $\mu \mathrm{g} / \mathrm{ml}$. The viability and proliferation of preadipocytes and differentiated adipocytes were analysed by the MTT technique. Lipid accumulation was measured by oil red O staining. The amount of triglycerides and glycerol released in the medium was analysed by a colorimetric method. Compared with the control, Jatupalathika extracts reduced fat accumulation (3.5 folds at $100 \mu \mathrm{g} / \mathrm{ml}$ ) and triglyceride content ( 3 folds at $100 \mu \mathrm{g} / \mathrm{ml}$ ), but increased glycerol release $(4.5$ folds at $10 \mu \mathrm{g} / \mathrm{ml})$. These activities could be attributed to the synergy between gallic acid and other bioactive components in the formula. Moreover, Jatupalathika extract $(100 \mu \mathrm{g} / \mathrm{ml})$ decreased the \% viability of both preadipocytes and differentiated adipocytes by $28.85 \pm 1.87 \%$ and $32.36 \pm 2.66 \%$, respectively. It could be concluded that Jatupalathika extract effectively reduced fat accumulation by the inhibition of lipogenesis and adipogenesis and the induction of lipolysis, which could be related to the induction of cell apoptosis or a reduction in cell proliferation. This herbal formula might potentially be used in the prevention and treatment of obesity. However, further investigations on mechanism of action, in vivo effects, and toxicity of the formula need to be done.

KEYWORDS: anti-obesity, Jatupalathika, 3T3-L1 adipocytes, lipogenesis, lipolysis

\section{INTRODUCTION}

Obesity, a chronic metabolic disorder caused by eating behaviours and an energy imbalance, has become a major health problem in humans worldwide. It is a risk factor associated with the progress of hyperlipidaemia, coronary heart disease, type II diabetes, hypertension, and metabolic syndromes $[1,2]$ as well as certain types of cancer and osteoarthritis [3]. Although several antiobesity drugs have been approved and marketed, most of them have been withdrawn due to their risk of severe side effects, such as heart valve damage and psychiatric disorders [4]. Even orlistat, a gastrointestinal lipase inhibitor used to help lose weight due to its safety, also causes flatulence, urgent bowel movements, oily stools, and non-negligible side effects [5].

Alternative ways to manage obesity are uses of bioactive compounds from plants. Various herbs, such as Fucus vesiculosis, Gymnema sylvestre, Pterocarpus marsupium, Commiphora mukul, and Antheraea perny, were reported for the treatment of obesity $[6,7]$. Nepeta japonica Maximiwicz was found to inhibit triglyceride accumulation and induce lipolysis in 3T3-L1 adipocytes [3]. Similarly, treatment of 3T3-L1 cells with Momordica charantia extract resulted in a de- crease of lipid accumulation and intracellular triglyceride amounts as well as a reduction in preadipocyte proliferation [8]. In an in vivo study, an aqueous extract of Cyperus rotundus L. tubers exhibited a potent anti-obesity activity in rats fed with a high-fat diet [9].

Numerous dietary supplements or nutraceuticals for weight control, including vitamins, amino acids, single herbs, herb formulas, or a combination of these compounds, have been launched in pharmacy stores and on websites of online selling anti-obesity products with weight loss guarantee [10]. However, important limitations which have hindered the acceptance of using these products are their scientific verification and quality control.

Jatupalathika (JP) is a traditional formulation of four dried fruits of Terminalia bellerica (Gaertn.) Roxb, Terminalia chebula Retz. var. chebula, and Terminalia arjuna Roxb. of the Combretaceae family, and Phyllanthus emblica L. from Euphorbiaceae. Our search revealed that JP formulations, e.g., pills, capsules, and solutions, were launched on the herbal market and used for various indications such as detoxification, anti-ageing, cellulite reduction, body fat depletion, and weight loss. However, scientific information for the JP formula related to reducing fat accumulation and anti- 
obesity still needs to be approved.

The pharmacological activities of the four individual herbs in the JP formula were investigated. T. bellerica (TB) extract has been reported to have antioxidation and hypoglycaemic effects, immunostimulating and anti-allergy activities, and the ability to lower total cholesterol, triglycerides, and uric acid [11]. Moreover, TB exhibited an increase in PPAR- $\alpha$ and PPAR- $\gamma$ and induced the transportation of glucose into cells by increasing the adipocyte proliferation [12]. Bark and leave extracts of T. arjuna (TA) were reported on having pharmacological activities such as hypoglycaemic and anti-diabetic effects in mice. However, the active compounds responsible for these activities remain unknown [11]. The extracts from fruits, leaves, and bark of T. chebula (TC) have been used as herbal medicines for carminatives, astringents, and phlegm removal treatment, as an anti-oxidant and anti-diabetic, and to lower blood cholesterol levels [13]. One previous study reported that $P$. emblica (PE) slightly affected the differentiation of adipocytes and could induce glucose uptake into cells. However, the expression of glut- 4 and transcription factors related to adipogenesis should be confirmed [14]. Interestingly, a clinical study comparing PE with simvastatin in a patient with hyperlipidaemia type II indicated that PE significantly lowered blood lipid in association with the antihypertensive drug [15]. Triphala is a traditional herbal formulation, commonly found in the herbal market, which consists of three of the four herbs in JP, TB, TC, and PE. The development of analytical techniques of Triphala for quality control was reported. Gallic acid, which was the major compound in Triphala, was generally used as a marker. However, the anti-obesity-related activity of JP has rarely been studied. Based on the literature review, we hypothesised that JP would have anti-obesity-related activities. This study aimed to determine the in vitro anti-obesity effects of JP formula compared with its individual herbs and its major components (gallic acid and ellagic acid). The scientific confirmation of the effect of JP extract on fat accumulation and lipolysis might be beneficial for the prevention and treatment of obesity.

\section{MATERIALS AND METHODS}

\section{Chemicals and reagents}

Dulbecco's modified Eagle medium (DMEM containing high glucose, L-glutamine, sodium pyruvate), insulin (INS), penicillin-streptomycin solution, dexamethasone (DEX), 3-isobutyl-1-methyl xanthine (IBMX), MTT [3-(4,5-dimethylthiazol-2-yl)-2,5diphenyl-tetrazolium bromide], gallic acid, ellagic acid, Oil Red O solution, phosphate-buffered saline (PBS), dimethyl sulphoxide (DMSO), triglyceride (TG), and glycerol assay kits were obtained from Sigma (MO, USA). Foetal bovine serum (FBS) was purchased from GIBCO (Invitrogen, NY, USA). All solvents and reagents were of analytical grade.

\section{Cell culture}

3T3-L1 cells, obtained from the American Type Culture Collection (ATCC, Rockville, MD, USA), were grown in culture plates containing DMEM supplemented with 10\% heat-inactivated FBS, 100 units/ml penicillin, and $100 \mu \mathrm{g} / \mathrm{ml}$ streptomycin. The cultures were kept at $37^{\circ} \mathrm{C}$ in a humidified chamber with $5 \% \mathrm{CO}_{2}$. The medium was changed every 2 days.

\section{Differentiation of 3T3-L1 cells to adipocytes}

The 3T3-L1 preadipocytes were plated in 24-well plates $\left(3 \times 10^{4}\right.$ cells/well) and cultured in DMEM containing $25 \mathrm{mmol} / 1$ glucose, $1 \mathrm{mmol} / 1$ sodium pyruvate, $4 \mathrm{mmol} / 1 \mathrm{~L}$-glutamine, $10 \%$ heat-inactivated FBS, $100 \mathrm{U} / \mathrm{ml}$ penicillin, and $100 \mu \mathrm{g} / \mathrm{ml}$ streptomycin at $37^{\circ} \mathrm{C}$ in $5 \% \mathrm{CO}_{2}$. After cell confluence (day 0 ) for 2 days, the medium was removed and a differentiation medium, containing $0.5 \mathrm{mmol} / 1 \mathrm{IBMX}, 2.5 \mu \mathrm{mol} / 1$ $\mathrm{DEX}$, and $10 \mu \mathrm{g} / \mathrm{ml}$ insulin INS, was added to induce cell differentiation. Test samples were added to the culture medium every 2 days. By day 12, more than $90 \%$ of the cells had differentiated into rounded cells with lipid droplets. Cells and culture medium were collected for further assays.

\section{Preparation of herbal test samples}

Dried fruits of T. bellerica (TB), T. arjuna (TA), T. chebula (TC), and P. emblica (PE) were cleaned and ground into powder. TB, TA, TC, and PE at a ratio of $1: 1: 1: 1$ by weight were mixed to prepare the Jatupalathika (JP) formula. $80 \mathrm{~g}$ each of JP, TB, TA, TC, and PE were macerated in $200 \mathrm{ml}$ distilled water for $4 \mathrm{~h}$ with occasional stirring and, then, sonicated. The supernatant was collected and filtered. The filtrate was dried in a hot air oven at $40^{\circ} \mathrm{C}$ for 2 days. The aqueous solution of JP, TB, TA, TC, and PE extracts, gallic acid (GA), and ellagic acid (EA) of different concentrations (10, 25, 50, $100 \mu \mathrm{g} / \mathrm{ml}$ ) and isoproterenol (Isop) $(10 \mu \mathrm{M})$ were prepared by dissolving each powder in distilled water. Test solutions of JP, TB, TA, TC, PE, GA, EA, and Isop were assayed and compared with the control (medium with cells, without test sample).

\section{Determination of gallic acid (GA) and ellagic acid (EA) by HPLC analysis}

HPLC analysis of GA and EA in JP, TB, TA, TC, PE extracts was performed. The HPLC system consisted of a LC-20AT pump, a SPD-20A UV detector equipped with a SPD-20A system controller, and a SIL-10ADVP sample injector fitted with a $20 \mu l$ sample loop (Shimadzu, Kyoto, Japan). The chromatographic separations were performed on a C18 column $(250 \mathrm{~mm} \times 4.6 \mathrm{~mm}$ i.d., $5 \mu \mathrm{m}, 250 \AA)\left(\mathrm{ACE}^{\circledR}\right.$, Scotland). The mobile phase was water-acetonitrile $(90: 10, \mathrm{v} / \mathrm{v})$ containing $1.0 \%$ 
v/v orthophosphoric acid, $\mathrm{pH}$ 3.00. All separations were performed isocratically at a flow rate of $1 \mathrm{ml} / \mathrm{min}$. The column temperature was maintained at room temperature $\left(27 \pm 2{ }^{\circ} \mathrm{C}\right)$. The peaks were determined using a UV detector set at a wavelength of $210 \mathrm{~nm}$. Standard solutions of GA and EA were prepared in the mobile phase at concentrations between $1-100 \mu \mathrm{g} / \mathrm{ml}$. The dried extracts were dissolved in the mobile phase. Standard solutions or test samples were injected directly into the system after filtering through a filter paper and a $0.45 \mathrm{~mm}$ membrane filter (Millipore, MA, USA). Identification of the components was based on comparing retention times of the peaks of the reference standards. Quantification of GA and EA was accomplished using the calibration curve of pure standards $\left(R^{2}=0.999\right)$.

\section{Cell viability assay}

The viability of 3T3-L1 adipocytes was assayed by the MTT technique according to the method of Mosmann [16]. For post-confluent preadipocytes, cells $\left(3 \times 10^{4}\right.$ cells $)$ were seeded in each well of a 24 -well plate and grown at $37^{\circ} \mathrm{C}$ in a humidified chamber with $5 \% \mathrm{CO}_{2}$ for $24 \mathrm{~h}$. Test solutions $(100 \mu \mathrm{l})$ were added to the cultures, and cell viability was assayed $24 \mathrm{~h}$ after the addition. For the viability of differentiated cells, post-confluent preadipocytes were treated with the test solutions $(100 \mu \mathrm{l})$ in a differentiation medium every 2 days for 12 days; after that, the viability was assayed. Then, $5 \mathrm{mg} / \mathrm{ml}$ MTT solution in phosphate-buffered saline (PBS, pH 7.4) (10 $\mu \mathrm{l})$ was added to each well. After $30 \mathrm{~min}$, the culture medium was discarded, and a DMSO:ethanol (1:1) solution $(200 \mu \mathrm{l})$ was added and mixed. The absorbance was measured at $595 \mathrm{~nm}$ using a microplate reader (Eon, BioTek Instruments, USA). \% Viability was calculated as follows:

$$
\% \text { viability }=\left[\left(\mathrm{Abs}_{\text {sample }}\right) /\left(\mathrm{Abs}_{\text {control }}\right)\right] \times 100
$$

\section{Determination of lipid accumulation by Oil Red $O$} staining assay

The lipid content in the adipocytes was determined using the Oil Red $\mathrm{O}$ staining assay as previously described [3]. Post-confluent preadipocytes $(3 \times$ $10^{4}$ cells) were grown as mentioned in the viability assay. Adipocytes were cultured in the differentiation medium and treated with either test samples or a vehicle (control) for 12 days with the medium changed every 2 days. On day 12, cells were washed with PBS solution and fixed in fresh 10\% formaldehyde for $1 \mathrm{~h}$. After that, cells were washed with $60 \%$ isopropanol, stained with $0.6 \%$ Oil Red O dye in $60 \%$ isopropanol for $15 \mathrm{~min}$, and then washed with distilled water 3 times. Red stained fat droplets were extracted from cells by adding isopropanol $(100 \mu \mathrm{l})$ to each well and mixing. The absorbance at $500 \mathrm{~nm}$ was measured using a microplate reader to calculate the fat accumulation.

\section{Determination of cellular triglyceride (TG) content}

Total cellular TG content was determined by a coupled enzyme assay. Post-confluent preadipocytes $(3 \times$ $10^{4}$ cells) were grown and cultured in the differentiation medium treated with test solution as described in the viability assay. The culture of differentiated adipocytes treated with test solution at day 12 was washed gently with PBS solution twice, and then lipid extraction buffer $(100 \mu \mathrm{l})$ was added to the cells. The plates were sealed, incubated at $90^{\circ} \mathrm{C}$ for $30 \mathrm{~min}$, cooled to room temperature, and then shaken for $1 \mathrm{~min}$ to mix the solution. TG content in the cloudy solution was analysed using a commercial TG assay kit according to the manufacturer's instructions. The colorimetric product, which was proportional to the TG content, was measured by absorbance at $570 \mathrm{~nm}$ using a microplate reader to calculate the TG content.

\section{Determination of glycerol content}

Lipolysis was assessed by the measurement of glycerol content released into the medium according to the manufacturer's instructions. Post-confluent preadipocytes $\left(3 \times 10^{4}\right.$ cells) were grown and cultured in the differentiation medium. The differentiated adipocytes in serum-free medium were treated with the sample extracts for $24 \mathrm{~h}$. The medium $(10 \mu \mathrm{l})$ was collected and mixed with a master reaction mix $(100 \mu \mathrm{l})$ in a 96-well plate. Plates were sealed and incubated at room temperature for $20 \mathrm{~min}$. The colorimetric product, which was proportional to the glycerol content, was measured by absorbance at $570 \mathrm{~nm}$ using a microplate reader to calculate the glycerol content.

\section{Statistical analyses}

Statistical analyses were conducted by one-way ANOVA with a post-hoc test, and $p<0.05$ was regarded as statistically significant.

\section{RESULTS AND DISCUSSION}

\section{Preparation of herbal test samples}

JP is a herbal mixture composed of TB, TA, TC, and PE fruit powder. The powder of individual herbs or the herbal mixture of JP was macerated in distilled water. JP aqueous extract of approximately 4\% (w/w of dried weight) was obtained. The physical appearance of JP was a brown viscous extract. The percentage yields of TB, TA, TC, and PE were 3.20, 3.44, 3.08, 2.97\% (w/w of dried weight), respectively; and the physical appearance was a greenish brown viscous extract.

\section{Determination of GA and EA by HPLC analysis}

GA and EA in JP and the four fruit extracts (TB, TA, $\mathrm{TC}$, and $\mathrm{PE}$ ) in the JP formula were determined by HPLC analysis. The retention time of GA and EA was approximately 4.4 and $3.9 \mathrm{~min}$, respectively. By comparing retention times of the extract peaks to the standard GA peak (Fig. 1), the GA content in the 


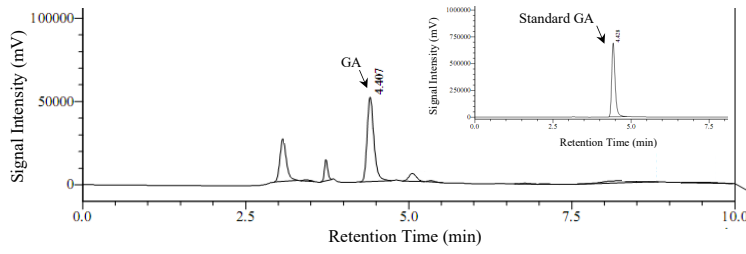

Fig. 1 HPLC chromatogram of Jatupalathika (JP) extract showing gallic acid (GA) as the major constituent.

Table 1 Determination of gallic acid in Jatupalathika and its four fruit extracts by HPLC analysis.

\begin{tabular}{lc}
\hline Extract & $\begin{array}{c}\text { Gallic acid } \\
\text { (mg/g of extract) }\end{array}$ \\
\hline Jatupalathika (JP) & $211.56 \pm 0.121$ \\
Terminalia bellerica (Gaertn.) Roxb (TB) & $207.48 \pm 0.145$ \\
Terminalia arjuna Roxb. (TA) & $181.66 \pm 0.041$ \\
Terminalia chebula Retz. var. chebula (TC) & $198.16 \pm 0.058$ \\
Phyllanthus emblica L. (PE) & $306.14 \pm 0.053$ \\
\hline
\end{tabular}

extracts was calculated, and the results are presented in Table 1. The results indicated that GA was one of the major polyphenolic compounds of JP and the four fruit extracts. The JP extract contained GA content of $211.56 \pm 0.121 \mathrm{mg} / \mathrm{g}$ of extract. Among the four fruit extracts, PE contained the highest GA content of $306.14 \pm 0.053 \mathrm{mg} / \mathrm{g}$ of extract. However, the EA content in all extracts was below the detection limit of the HPLC analysis and could not be clearly identified in the chromatogram.

\section{Cell viability assay}

The 3T3-L1 cell line, generally used as a model of adipocyte differentiation and fat accumulation, was used in this investigation. The differentiation of preadipocytes into adipocytes is an important step to obtain mature adipocytes, which possess the capability to take up, synthesise, and store lipids [17]. The viability of 3T3-L1 preadipocytes and differentiated

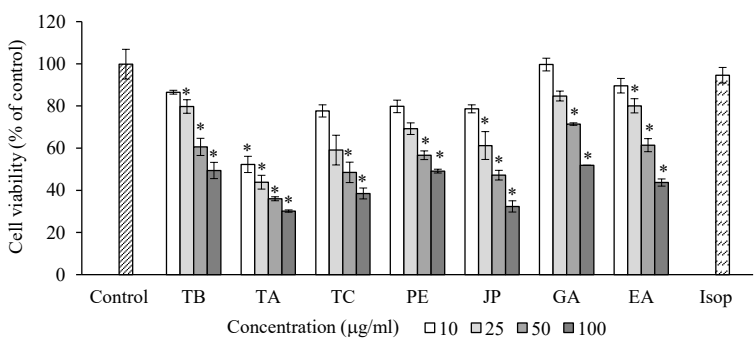

Fig. 2 Effect of T. bellerica (TB), T. arjuna (TA), T. chebula (TC), P. emblica (PE), Jatupalathika (JP) extracts, gallic acid (GA), ellagic acid (EA), and isoproterenol (Isop) on cell viability of 3T3-L1 adipocytes. Values are expressed as mean $\pm \mathrm{SD}(n=3),{ }^{*} p<0.05$ as compared with the untreated control.

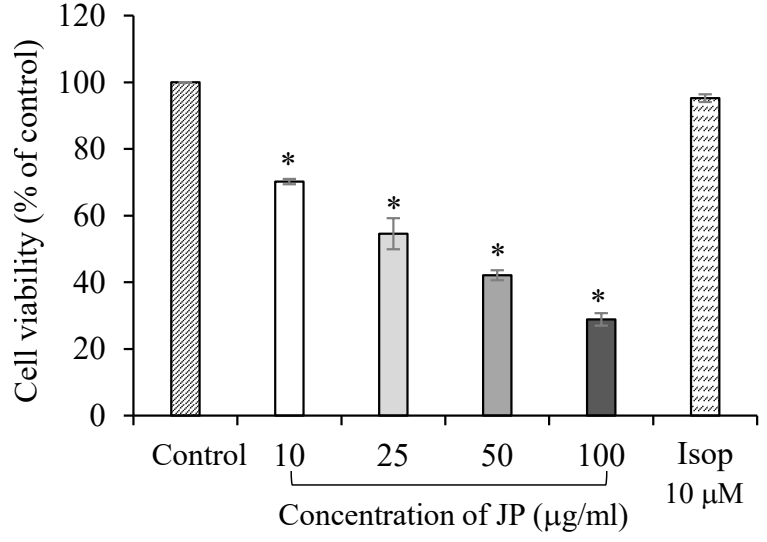

Fig. 3 Effect of Jatupalathika (JP) extract and isoproterenol (Isop) on cell viability of 3T3-L1 preadipocytes. Values are expressed as mean \pm SD $(n=3),{ }^{*} p<0.05$ as compared with the untreated control.

adipocytes treated with JP extract, four herbal ingredients of JP formula (TB, TA, TC, and PE), GA, and EA was determined using the MTT assay, and the results were compared with the control. The mitochondrial reduction of MTT to formazan is directly proportional to the living cell number [16]. The effect of the tested samples on the \% viability of differentiated adipocytes is shown in Fig. 2. At the concentration of $10 \mu \mathrm{g} / \mathrm{ml}$, JP extract did not cause any alteration in viable cell numbers of differentiated adipocytes, while JP at 25$100 \mu \mathrm{g} / \mathrm{ml}$ showed an inhibitory effect on cell viability in a dose-response relationship with the lowest \% viability of $32.36 \pm 2.66$ (at $100 \mu \mathrm{g} / \mathrm{ml}$ ). TB at 25$100 \mu \mathrm{g} / \mathrm{ml}$, TA at 10-100 $\mu \mathrm{g} / \mathrm{ml}$, and TC and PE at 50 and $100 \mu \mathrm{g} / \mathrm{ml}$ significantly decreased the adipocyte viability in a dose-dependent manner. These individual herbal extracts at $100 \mu \mathrm{g} / \mathrm{ml}$ exhibited the lowest percentages of viable cell number of $30.16 \pm 0.54 \%$, $38.51 \pm 2.59 \%, 49.11 \pm 0.90 \%$, and $49.40 \pm 3.85 \%$ for TA, TC, PE, and TB, respectively. For the major compounds in JP extract and the four individual plants, GA at 10 and $25 \mu \mathrm{g} / \mathrm{ml}$ and EA at $10 \mu \mathrm{g} / \mathrm{ml} \mathrm{did}$ not affect cell viability. However, GA and EA at the concentration of $100 \mu \mathrm{g} / \mathrm{ml}$ significantly reduced the viability of differentiated adipocytes by $51.83 \pm 0.05 \%$ and $43.69 \pm 1.70 \%$, respectively. This was similar to the results for the four individual herbs and the JP extract. Isoproterenol did not cause any alteration in the viable cell number. The effect of JP extract on cell viability of 3T3-L1 preadipocytes was also investigated. As shown in Fig. 3, JP at 10-100 $\mu \mathrm{g} / \mathrm{ml}$ decreased preadipocyte viability in a dose-dependent manner with the lowest viability being $28.85 \pm 1.87 \%$ (at $100 \mu \mathrm{g} / \mathrm{ml}$ ).

A reduction in the number of viable adipocytes due to treatment with JP, TA, TC, PE, TB extracts, GA, and EA may contribute to the induction of cell apoptosis 


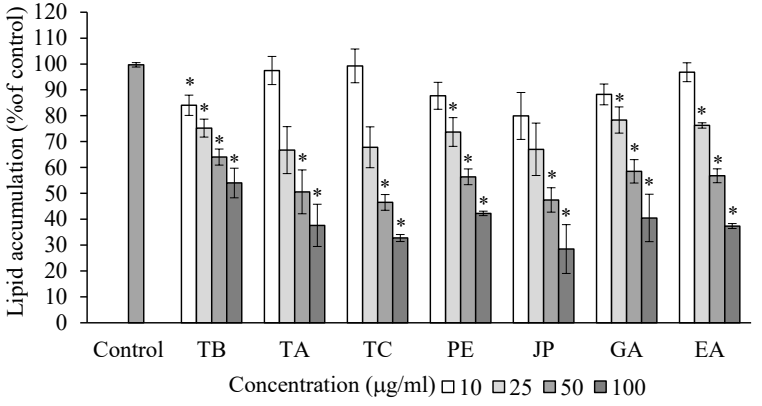

Fig. 4 Effects of T. bellerica (TB), T. arjuna (TA), T. chebula (TC), and P. emblica (PE), Jatupalathika (JP) extracts, gallic acid (GA), and ellagic acid (EA) on lipid accumulation in 3T3$\mathrm{L} 1$ adipocytes by Oil Red $\mathrm{O}$ staining technique. Values are expressed as mean $\pm \mathrm{SD}(n=3),{ }^{*} p<0.05$ as compared with the untreated control.

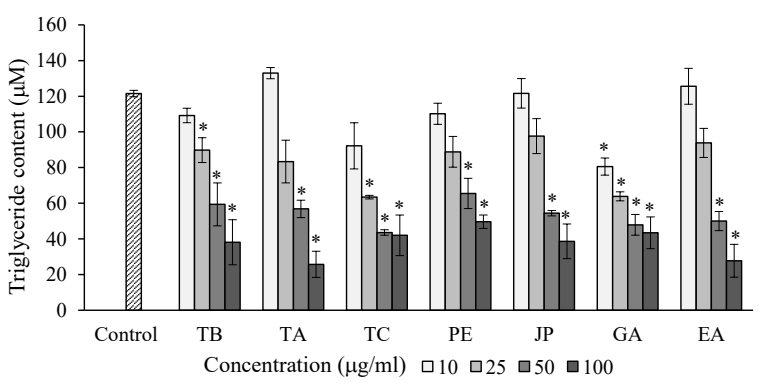

Fig. 5 Effects of T. bellerica (TB), T. arjuna (TA), T. chebula (TC), and P. emblica (PE), Jatupalathika (JP) extracts, gallic acid (GA), and ellagic acid (EA) on triglyceride content in 3T3-L1 adipocytes. Values are expressed as mean \pm SD $(n=$ 3), ${ }^{*} p<0.05$ as compared with the untreated control.

or the suppression of cell viability and proliferation. Moreover, the JP extract affected both differentiated adipocytes and preadipocytes in the same way. These could be potential mechanisms for reducing adipogenesis.

\section{Lipid accumulation assay}

The adipocyte volume mainly depends on the accumulation of triacylglycerols. The differentiated adipocytes were stained with Oil Red O solution and lipid contents in 3T3-L1 adipocytes undergoing adipogenesis were measured spectrophotometrically. The untreated differentiated adipocytes contained many lipid droplets with the Oil Red O dye staining, indicating lipid accumulation. The accumulation was significantly reduced by higher doses of JP, the four individual herb extracts, GA, and EA. The effects of the tested samples on intracellular lipid accumulation in 3T3-L1 adipocytes are shown in Fig. 4. The inhibition of lipid accumulation was observed by the treatment of JP at 50 and $100 \mu \mathrm{g} / \mathrm{ml}$, TB at $10-100 \mu \mathrm{g} / \mathrm{ml}$, TA and TC at 50, $100 \mu \mathrm{g} / \mathrm{ml}, \mathrm{PE}$ at $25-100 \mu \mathrm{g} / \mathrm{ml}$, and GA and EA at 25-

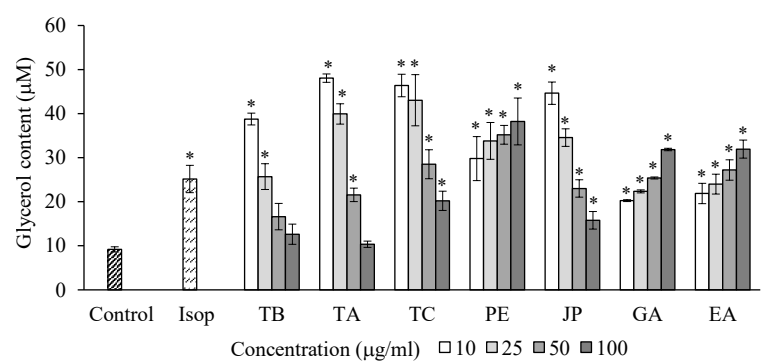

Fig. 6 Effects of T. bellerica (TB), T. arjuna (TA), T. chebula (TC), P. emblica (PE), Jatupalathika (JP) extracts, gallic acid (GA), ellagic acid (EA), and isoproterenol (Isop) on glycerol release in 3T3-L1 adipocytes. Values are expressed as mean $\pm \operatorname{SD}(n=3),{ }^{*} p<0.05$ as compared with the untreated control.

$100 \mu \mathrm{g} / \mathrm{ml}$ with the maximum inhibition at $100 \mu \mathrm{g} / \mathrm{ml}$. JP extract exhibited the highest \% inhibition of lipid accumulation of about $71.50(28.50 \pm 9.43 \%$ of control, $100 \mu \mathrm{g} / \mathrm{ml}$ ), which was higher than that of the four individual herbs with the maximum \% inhibition being; TC 67.25 (32.75 $\pm 1.35 \%$ of control) $>$ TA 62.35 (37.65 $\pm 8.16 \%$ of control) $>$ PE 57.73 $(42.27 \pm 0.86 \%$ of control) $>$ TB 45.99 (54.01 $\pm 5.72 \%$ of control). These results were similar to those for GA 67.25 (32.75 $\pm 1.35 \%$ of control), and EA 62.35 (37.65 $\pm 8.16 \%$ of control).

Lipid accumulation based on the triglyceride (TG) content of 3T3-L1 cells was determined by a colorimetric assay. The effects of the tested samples on intracellular TG content in 3T3-L1 adipocytes are shown in Fig. 5. The result showed that JP at 50 and $100 \mu \mathrm{g} / \mathrm{ml}$, TB and TC at 25-100 $\mu \mathrm{g} / \mathrm{ml}$, TA and $\mathrm{PE}$ at 50 and $100 \mu \mathrm{g} / \mathrm{ml}, \mathrm{GA}$ at $10-100 \mu \mathrm{g} / \mathrm{ml}$, and EA at 50 and $100 \mu \mathrm{g} / \mathrm{ml}$ caused a decrease in TG content in adipocytes, with the maximum inhibition seen at $100 \mu \mathrm{g} / \mathrm{ml}$. TG deposition in adipocytes was decreased following treatment with JP extract by about $24.15 \pm 5.72 \mu \mathrm{M}$, with a maximum $\%$ inhibition of 80.12. Similarly, TG content from the treatment of TA, TB, TC, and PE was approximately 25.76 \pm 7.33 , $38.13 \pm 12.63,42.01 \pm 11.37$, and $49.64 \pm 3.76 \mu \mathrm{M}$, respectively, with the maximum \% inhibition being 78.80 , 68.62, 65.42, and, 59.14, respectively. Both GA and EA significantly decreased the TG content to $19.93 \pm 8.90$ and $27.75 \pm 9.21 \mu \mathrm{M}$ with the highest $\%$ inhibition of 83.60 and 77.16, respectively.

Our findings indicate that JP extract significantly reduced lipogenesis via the inhibition of fat accumulation and TG content in adipocytes. Nevertheless, GA and EA reduced fat accumulation less than JP extract. These results indicate that GA may be partially responsible for this activity together with other phytocompounds in the formula. Although EA has beneficial effects on fat and triglyceride reduction within adipocytes, its impact might be low due to the 
low or negligible amount of EA in the extracts. For TG content, GA may be responsible for this activity since it caused a reduction in TG content similar to the JP extract. The four individual herbs in the JP formula also exhibited inhibition of fat accumulation and a decrease in TG content in adipocytes, but their effects were lower than that of the JP extract. This investigation indicated that the four individual herbs in the JP formula have a synergistic effect in the reduction of lipogenesis. Moreover, these may be partially due to a decrease in cell numbers resulting from the inhibition of cell viability and the proliferation of adipocytes. For further study, a positive control such as Troglitazone, a known PPAR $\gamma$ agonist, might be used to clarify the related mechanism of action for fat accumulation [18].

The effect of the JP extract and its herbal mixtures on lipolysis was evaluated by the measurement of glycerol released into the culture medium of 3T3-L1 adipocytes using a colorimetric assay. Isoproterenol was used as a positive control to induce lipolysis resulting in glycerol and free fatty acid releases [19]. The effects of the tested samples on glycerol release from 3T3-L1 adipocytes are shown in Fig. 6. JP extract at $10-50 \mu \mathrm{g} / \mathrm{ml}$, similar to TA (10-50 $\mu \mathrm{g} / \mathrm{ml})$, TC (10-100 $\mu \mathrm{g} / \mathrm{ml})$, TB (10 and $25 \mu \mathrm{g} / \mathrm{ml})$, and PE (10-100 $\mu \mathrm{g} / \mathrm{ml})$, significantly increased glycerol release. The maximum contents of detected glycerol were as follows: JP $(44.63 \pm 2.53 \mu \mathrm{M}$, $10 \mu \mathrm{g} / \mathrm{ml})$, TA $(48.05 \pm 0.95 \mu \mathrm{M}, 10 \mu \mathrm{g} / \mathrm{ml})$, TC $(46.38 \pm 2.55 \mu \mathrm{M}, 10 \mu \mathrm{g} / \mathrm{ml})$, TB $(38.77 \pm 1.34 \mu \mathrm{M}$, $10 \mu \mathrm{g} / \mathrm{ml})$, and PE $(38.22 \pm 5.32 \mu \mathrm{M}, 100 \mu \mathrm{g} / \mathrm{ml})$. However, glycerol releases following treatment with JP, TB, TA, and TC extracts at higher concentrations tended to decline. This may be due to the inhibitory effect of the extracts on cell viability. All concentrations of GA and EA (10-100 $\mu \mathrm{g} / \mathrm{ml})$ significantly increased glycerol release with the maximum amount of $31.86 \pm 0.28 \mu \mathrm{M}$ and $31.95 \pm 0.28 \mu \mathrm{M}(100 \mu \mathrm{g} / \mathrm{ml})$, respectively. For isoproterenol, the elevated glycerol content of $25.16 \pm 3.10 \mu \mathrm{M}$ was detected.

Fat accumulation is determined by the balance between lipogenesis and lipolysis. Lipogenesis includes the syntheses of fatty acids and triglycerides taking place in the liver and adipose tissues, while adipogenesis involves the differentiation of preadipocytes into mature fat cells [20]. The inhibition of adipocyte differentiation is an important strategy to control obesity since an increase in adipose mass is caused by both adipocyte hypertrophy and adipocyte hyperplasia [21].

In this study, we found that JP extract and the four herbal components, TB, TA, TC, and PE, had anti-obesity activity related to lipogenesis, adipogenesis, and lipolysis in 3T3-L1 adipocytes. However, the mechanisms pertaining to these effects, such as the expression of key adipogenic transcription factors including peroxisome proliferator-activated receptor gamma (PPAR $\gamma$ ) and CCAAT/enhancer-binding pro- tein (C/EBP $\alpha$ ) [21], still need to be explored. Further evaluation of toxicity and in vivo experiments is necessary to be undertaken to support its therapeutic uses.

Various strategies have been used for weight management and obesity, such as monitoring of food intake and calories, increased exercise, and behavioural changes involving diet. The inhibition of lipogenesis, the stimulation of lipolysis, and the induction of adipocyte apoptosis result in a reduction in adipose tissue mass. A reduction of carbohydrate or fat absorption and the inhibition of preadipocyte proliferation and differentiation have been assumed to account for the mechanisms of anti-obesity [22]. Many investigations have indicated that novel substances from natural sources affect adipocyte differentiation and gene regulation associated with obesity [23]. The potential of herbal formulations presenting anti-obesity activities was also reported. KBH-1, mixtures of Polygala tenuifolia, Curcuma longa, and Saururus chinensis [24], and SH21B composed of seven herbs [25] inhibited lipid accumulation in 3T3-L1 adipocytes and in mice with HFD-induced obesity. Although the mechanisms of action of many traditional herbal medicines are not clear, several herbal formulas are more efficacious than an equivalent dose of their individual components alone [26]. In some cases, the isolated phytocompounds may be effective only in combination with other components in the mixture due to synergistic effects [27]. Developing an effective anti-obesity agent derived from herbal formulations is therefore a tremendous challenge for researchers.

\section{CONCLUSION}

In conclusion, our results demonstrated that the Jatupalathika extract efficiently inhibited fat accumulation by the inhibition of adipogenesis, lipogenesis, and the induction of lipolysis in 3T3-L1 adipocytes in a dose-response relationship. Jatupalathika extract was more effective than an equivalent dose of its individual herbal ingredients and its major components, the gallic acid and the ellagic acids. These findings suggested that Jatupalathika formula. This herbal formula might potentially be used in the prevention and treatment of obesity.

Acknowledgements: The authors gratefully acknowledge financial support from the Agricultural Research Funding Agency of Thailand (Public Organization), Thailand (grant no. CRP6305030010).

\section{REFERENCES}

1. Kopelman PG (2000) Obesity as a medical problem. Nature 404, 635-641.

2. Leonhardt M, Hrupka B, Langhans W (1999) New approaches in the pharmacological treatment of obesity. Eur J Nutr 38, 1-13.

3. Roh C, Jung U (2012) Nepeta japonica Maximowicz extract from natural products inhibits lipid accumulation. J Sci Food Agr 92, 2195-2199. 
4. Kang JG, Park C (2012) Anti-obesity drugs: A review about their effects and safety. Diabetes Metab $J$ 36, 13-25.

5. Bray GA, Tartaglia LA (2000) Medicinal strategies in the treatment of obesity. Nature 404, 672-677.

6. Goswami P, Khale A, Shah S (2011) Medicinal herbs and obesity: review. Int J Pharm Sci Rev Res 11, 69-74.

7. Zhu L, Guo G, Fan Z-Q, Wang N, Zou D-Q, Shi X-Q (2021) Alleviation of high-fat-diet induced obesity and cholesterol accumulation in mice by extracts from male zooid of Antheraea pernyi. ScienceAsia 47, 162-169.

8. Popovich DG, Li L, Zhang W (2010) Bitter melon (Momordica charantia) triterpenoid extract reduces preadipocyte viability, lipid accumulation and adiponectin expression in 3T3-L1 cells. Food Chem Toxicol 48, 1619-1626.

9. Athesh K, Divakar M, Brindha P (2014) Anti-obesity potential of Cyperus rotundus L. aqueous tuber extract in rats fed on high fat cafeteria diet. Asian J Pharm Clin Res 7, 88-92.

10. Banjare J, Bhalerao S (2016) A survey of marketed Ayurvedic/herbal anti-obesity products. Int $J$ Pharm Pharm Sci 8, 384-386.

11. Subramoniom A (2016) Anti-diabetes mellitus plants. In: Subramoniom A (ed) Plants with Anti-diabetes Mellitus Properties, CRC Press, London, pp 400-402.

12. Yang MH, Vasquez Y, Ali Z, Khan IA, Khan SI (2013) Constituents from Terminalia species increase PPAR $\alpha$ and PPAR $\gamma$ levels and stimulate glucose uptake without enhancing adipocyte differentiation. $J$ Ethnopharmacol 16, 490-498.

13. Gupta PC (2012) Biological and pharmacological properties of Terminalia chebula Retz. (Haritaki): an overview. Int J Pharm Pharm Sci 4, 62-68.

14. Kalekar S, Karve A, Munshi R, Bhalerao S (2012) Evaluation of the adipogenic potential and glucose uptake stimulatory activity of Phyllanthus emblica and Tinospora cordifolia: an in vitro study. Int J Pharma Bio Sci 3, 230-236.

15. Zaki M, Begum W, Bhat TA, Kausar HS (2014) Amla (Emblica officinalis GAERTN) the wonderful unani drug: a review. World J Pharm Pharm Sci 3, 1560-1572.

16. Mosmann T (1983) Rapid colorimetric assay for cellular growth and survival: application to proliferation and cytotoxicity assays. J Immunol Methods 65, 55-63.

17. Wang YW, Jones PJH (2004) Conjugated linoleic acid and obesity control: efficacy and mechanisms. Int $J$ Obesity 28, 941-955.

18. Li KK, Liu CL, Shiu HT, Wong HL, Siu WS, Zhang C, Han XQ, Ye CX, et al (2016) Cocoa tea (Camellia ptilophylla) water extract inhibits adipocyte differentiation in mouse 3T3-L1 preadipocytes. Sci Rep 6, ID 20172.

19. Louis C, Van den Daelen C, Tinant G, Bourez S, Thome JP, Donnay I, Larondelle Y, Debier C (2014) Efficient in vitro adipocyte model of long-term lipolysis: a tool to study the behaviour of lipophilic compounds. In Vitro Cell Dev Biol Anim 50, 507-518.

20. Kersten S (2001) Mechanisms of nutritional and hormonal regulation of lipogenesis. EMBO Rep 2, 282-286.

21. Caro JF, Dohm LG, Pories WJ, Sinha MK (1989) Cellular alterations in liver, skeletal muscle, and adipose tissue responsible for insulin resistance in obesity and type II diabetes. Diabetes Metab Rev 5, 665-689.

22. Zhu H, Wang L, Wang X, Pan H, Li N, Yang H, Jin M, Zang $\mathrm{B}$, et al (2014) Hormone-sensitive lipase is involved in the action of hydroxysafflor yellow A (HYSA) inhibiting adipogenesis of 3T3-L1 cells. Fitoterapia 93, 182-188.

23. Yang Y, Yang X, Xu B, Zeng G, Tan J, He X, Hu C, Zhou Y (2014) Chemical constituents of Morus alba L. and their inhibitory effect on 3T3-L1 preadipocyte proliferation and differentiation. Fitoterapia 98, 222-227.

24. Lee J, Kim T, Lee J, Lee KJ, Kim H, Yun B, Jeon J, Kim SK, et al (2015) The herbal medicine KBH-1 inhibits fat accumulation in 3T3-L1 adipocytes and reduces high fat diet-induced obesity through regulation of the AMPK pathway. PLoS One 10, e0142041.

25. Lee H, Kang R, Yoon Y (2010) SH21B, an anti-obesity herbal composition, inhibits fat accumulation in 3T3L1 adipocytes and high fat diet-induced obese mice through the modulation of the adipogenesis pathway. $J$ Ethnopharmacol 17, 709-717.

26. Williamson EM (2001) Synergy and other interactions in phytomedicines. Phytomedicine 8, 401-409.

27. Andersen C, Rayalam S, Della-Fera MA, Baile CA (2010) Phytochemicals and adipogenesis. Biofactors 36, 415-422. 